\title{
A DIFFERENTIAL EQUATIONS APPROACH TO HAMILTONIAN SYSTEMS
}

\author{
Dirk Fesser, Dirk Saller, Werner M. Seiler \\ Lehrstuhl für Mathematik I, Universität Mannheim, \\ 68131 Mannheim, Germany \\ (Received \\ 2001)
}

We present an intrinsic definition of a (possibly time-dependent) Hamiltonian differential equation as a submanifold of the first-order jet bundle over a fibred cosymplectic manifold. The equivalence of the standard constraint algorithm in mechanics to the completion procedure in differential equations theory is explicitly demonstrated. As an application, we study covariant classical mechanics. Finally, some problems with constraint algorithms in field theories are indicated.

Keywords: Hamiltonian system, differential equation, jet bundle, completion, formal integrability, cosymplectic manifold, constraint, covariant classical mechanics

\section{Introduction}

Hamiltonian systems are of great interest in mathematical physics. They exhibit a rich geometric structure and form the basis of quantisation. One typically has met them first in the form of a system of ordinary differential equations

$$
\frac{d q^{i}}{d t}=\frac{\partial H}{\partial p_{i}}, \quad \frac{d p_{i}}{d t}=-\frac{\partial H}{\partial q^{i}} .
$$

Here $H(t, q, p)$ is the Hamiltonian of the system, usually interpreted as the total energy. The functions $q^{i}(t)$ and $p_{i}(t)$ represent positions and momenta, respectively. Solutions of (1) describe trajectories of the system.

For autonomous systems, i.e. systems where $\partial H / \partial t=0$, symplectic geometry provides a convenient framework for an intrinsic description. One considers a $2 n$-dimensional manifold $\mathcal{M}$ equipped with a closed, non-degenerate 2 -form $\Omega$. Often, the equation

$$
\iota_{X_{H}} \Omega=d H
$$

is called the "equation of motion" of the system. Strictly speaking, this is not correct: (2) is an algebraic relation that associates a vector field $X_{H}$ on $\mathcal{M}$ with the function $H$; the equation of motion is the flow equation of this vector field. 
The goal of this article is to present a unified framework combining the differential equations approach of (1) with the intrinsic geometric approach of (2). Since time appears in (1), it is not surprising that such a framework automatically covers time-dependent systems, too. Thus on the geometric side we need cosymplectic instead of symplectic manifolds. For the intrinsic description of differential equations we use jet bundles and not tangent bundles. There are two reasons for this. Firstly, this is more natural for time-dependent systems and, secondly, we believe that jet bundles provide a better basis for a later generalisation to field theories.

Such a unified framework is particularly of interest for systems with constraints. It is well-known that such a system might contain hidden constraints which must be exhibited by a constraint algorithm. One of us has shown earlier that these algorithms correspond to the completion procedures in differential equations theory $[19,20,21]$. While the completion theory can also handle arbitrary systems of partial differential equations, we are not aware of any general constraint algorithm for field theories.

The geometric completion of ordinary differential equations within the tangent bundle formalism has already been studied by a number of authors. We mention [10, 11, 12] where the resulting theory has also been applied to constrained Hamiltonian systems. But also several numerical analysts have studied this problem under the name of differential algebraic equations [14, 15, 22].

The article is organised as follows. The next section briefly reviews the formal theory of differential equations. It contains a direct construction of the first-order jet bundle simplifying many constructions. Cosymplectic manifolds and their use for the modelling of time-dependent Hamiltonian systems are the topic of Sect. 3. The following section contains the main results of the article: we give an intrinsic definition of Hamiltonian differential equations as fibred submanifolds of the first-order jet bundle, show its equivalence to the classical approach and prove that the usual constraint algorithm coincides with the completion procedure for ordinary differential equations. As a larger example covariant classical mechanics is briefly studied in Sect. 5. Some problems of an extension of our results to field theories are discussed in Sect. 6. Finally, some conclusions are given.

\section{Differential Equations and Jet Bundles}

Jet bundles over fibred manifolds provide a natural geometric framework for studying differential equations [17]. Traditionally, they are introduced via truncated power series as equivalence classes of smooth sections. For our purposes, a different approach turns out to be more convenient.

Definition 1. Let $\pi: \mathcal{M} \rightarrow \mathcal{B}$ be a fibred manifold. The first-order jet bundle over $\mathcal{M}$ is the affine bundle $\pi_{0}^{1}: J_{1} \mathcal{M} \rightarrow \mathcal{M}$ whose fibre at the point $\xi \in \mathcal{M}$ with $\pi(\xi)=x$ is

$$
\left(J_{1} \mathcal{M}\right)_{\xi}=\left\{\lambda \in T_{x}^{*} \mathcal{B} \otimes T_{\xi} \mathcal{M} \mid T_{\xi} \pi \circ \lambda=\operatorname{id}_{T_{x} \mathcal{B}}\right\}
$$

This definition is related to the classical one as follows. Let $\sigma: \mathcal{B} \rightarrow \mathcal{M}$ be a section. It induces the section $j_{1} \sigma: \mathcal{B} \rightarrow J_{1} \mathcal{M}$ defined by $j_{1} \sigma(x)=\left(\sigma(x), T_{x} \sigma\right)$. Thus we may 
identify each point $(\xi, \lambda) \in J_{1} \mathcal{M}$ with the equivalence class of sections

$$
(\xi, \lambda) \cong\left\{\sigma: \mathcal{B} \rightarrow \mathcal{M} \mid \sigma \circ \pi(\xi)=\xi, T_{\pi(\xi)} \sigma=\lambda\right\}
$$

Let $\left(x^{i}, u^{\alpha}\right)$ be adapted local coordinates on $\mathcal{M}$. We may then use as coordinates on $J_{1} \mathcal{M}$ tuples $\left(x^{i}, u^{\alpha}, u_{i}^{\alpha}\right)$. The map $\lambda$ defined by the fibre component of the point $\left(x^{i}, u^{\alpha}, u_{i}^{\alpha}\right) \in J_{1} \mathcal{M}$ takes in these coordinates the form $\left(\dot{x}^{i}\right) \mapsto\left(\dot{x}^{i}, u_{i}^{\alpha} \dot{x}^{i}\right)$ where the $\left(\dot{x}^{i}\right)$ are induced coordinates in $T_{x} \mathcal{B}$ and where we have used the summation convention.

The geometry of the jet bundle is to a large extent determined by its contact structure. It can be described in many different ways. We use the contact map

$$
\Gamma_{1}:\left\{\begin{array}{c}
J_{1} \mathcal{M} \times_{\mathcal{B}} T \mathcal{B} \\
(\xi, \lambda, v) \quad T \mathcal{M} \\
\longmapsto(\xi, \lambda(v))
\end{array}\right.
$$

which in our approach becomes a simple evaluation map. In local coordinates, it is easier to describe $\Gamma_{1}$ as a map $J_{1} \mathcal{M} \rightarrow T^{*} \mathcal{B} \otimes T \mathcal{M}$ defined by $\left(x^{i}, u^{\alpha}, u_{i}^{\alpha}\right) \mapsto d x^{i} \otimes\left(\partial_{x^{i}}+u_{i}^{\alpha} \partial_{u^{\alpha}}\right)$.

An important property of the first-order jet bundle is that each section $\gamma: \mathcal{M} \rightarrow J_{1} \mathcal{M}$ defines a connection on the fibred manifold $\mathcal{M}$ (and vice versa). Its horizontal space at the point $\xi \in \mathcal{M}$ is $H_{\xi}=\Gamma_{1}\left(\gamma(\xi), T_{\pi(\xi)} \mathcal{B}\right)$. Alternatively, we use the vertical projector

$$
\mathcal{V}[\gamma]:\left\{\begin{aligned}
T \mathcal{M} & \longrightarrow \mathcal{M} \\
(\xi, w) & \longmapsto\left(\xi, w-\Gamma_{1}\left(\gamma(\xi), T_{\xi} \pi(w)\right)\right)
\end{aligned}\right.
$$

where $V \mathcal{M}=\operatorname{ker} T \pi$ denotes the vertical bundle. If the section $\gamma$ is locally written as $\left(x^{i}, u^{\alpha}\right) \mapsto\left(x^{i}, u^{\alpha}, g_{i}^{\alpha}(x, u)\right)$, then $\mathcal{V}[\gamma]\left(x^{i}, u^{\alpha} ; \dot{x}^{i}, \dot{u}^{\alpha}\right)=\left(x^{i}, u^{\alpha} ; 0, \dot{u}^{\alpha}-g_{i}^{\alpha}(x, u) \dot{x}^{i}\right)$.

We omit a rigorous introduction of the higher-order jet bundles. They can be constructed in several ways; for example one may naturally identify $J_{2} \mathcal{M}$ with a fibred subbundle of $J_{1}\left(J_{1} \mathcal{M}\right)$ and then iterate. For our purposes it suffices to mention that the higher-order jet bundles form a natural hierarchy $\pi_{q}^{r}: J_{r} \mathcal{M} \rightarrow J_{q} \mathcal{M}$ for $r>q$ and that each jet bundle is also a fibred manifold $\pi^{q}: J_{q} \mathcal{M} \rightarrow \mathcal{B}$.

Definition 2. A differential equation is a fibred submanifold $\mathcal{R}_{q} \subseteq J_{q} \mathcal{M}$. A solution is a section $\sigma: \mathcal{B} \rightarrow \mathcal{M}$ such that $\operatorname{Im}\left(j_{1} \sigma\right) \subseteq \mathcal{R}_{q}$.

Note that this definition does not distinguish between scalar equations and systems. Locally, such a submanifold can always be represented as the zero set of some system of equations which brings us back to the traditional picture of differential equations. Similarly, a section $\sigma: \mathcal{B} \rightarrow \mathcal{M}$ is locally of the form $\left(x^{i}\right) \mapsto\left(x^{i}, s^{\alpha}(x)\right)$. Hence its prolongation $j_{1} \sigma$ is of the local form $\left(x^{i}\right) \mapsto\left(x^{i}, s^{\alpha}(x),\left(\partial s^{\alpha} / \partial x^{i}\right)(x)\right)$ and we get the usual notion of a solution.

A special situation arises for first-order ordinary differential equations. If they are of the local form $\dot{u}^{\alpha}=g^{\alpha}\left(x^{i}, u^{\beta}\right)$, then we can write (at least locally) $\mathcal{R}_{1}=\operatorname{Im} \gamma$ for some section $\gamma: \mathcal{M} \rightarrow J_{1} \mathcal{M}$. Hence any such equation defines a connection.

Two natural operations with differential equations are projection and prolongation. The first one lowers the order, $\mathcal{R}_{q-r}^{(r)}=\pi_{q-r}^{q}\left(\mathcal{R}_{q}\right) \subseteq J_{q-r} \mathcal{M}$, whereas the second one 
raises it, $\mathcal{R}_{q+r}=J_{r} \mathcal{R}_{q} \cap J_{q+r} \mathcal{M}$. Projection is difficult to describe in local coordinates, as it requires the elimination of all derivatives of order greater than $q-r$. In contrast, prolongation means simply to differentiate all equations $r$ times.

One could be tempted to think that projection and prolongation are some kind of inverse operations, but this is not the case. If we first prolong a differential equation $\mathcal{R}_{q}$ to order $q+r$ and then project back to order $q$, we will obtain in general an equation $\mathcal{R}_{q}^{(r)}=\pi_{q}^{q+r}\left(\mathcal{R}_{q+r}\right) \subseteq \mathcal{R}_{q}$ which is only a submanifold of the original equation $\mathcal{R}_{q}$. This effect is due to the appearance of previously hidden integrability conditions. Such conditions represent obstructions to the construction of formal power series solutions.

Definition 3. The differential equation $\mathcal{R}_{q} \subseteq J_{q} \mathcal{M}$ is called formally integrable, if $\mathcal{R}_{q+r}^{(1)}=\mathcal{R}_{q+r}$ for all $r \geq 0$.

Formal integrability is in general difficult to check, as its definition poses infinitely many conditions. However, we will see below that for ordinary differential equations this is no problem. In contrast, for partial differential equations no one has yet been able to derive a finite criterion for formal integrability. One must use the stronger concept of an involutive system; for details on it we refer to the literature [5, 13, 18]. Here we only remark that every involutive system is formally integrable but not vice versa.

It is straightforward to show that formally integrable equations possess formal power series solutions which explains the name. Given an analytic equation, one can even prove that, for appropriate analytic initial data, the formal series converge. This is the Cartan-Kähler Theorem generalising the classical Cauchy-Kovalevskaya Theorem. Thus it is of advantage to deal with a formally integrable or even better an involutive system. Fortunately, this can always be achieved under some fairly mild regularity assumptions.

CARtan-Kuranishi TheORem 1. Let $\mathcal{R}_{q} \subseteq J_{q} \mathcal{M}$ be a sufficiently regular differential equation. There exist two finite integers $r, s \geq 0$ such that $\mathcal{R}_{q+r}^{(s)} \subseteq J_{q+r} \mathcal{M}$ is an equivalent involutive differential equation.

Here equivalent means that the formal solution spaces of the two equations are identical. Note that in general the involutive equation $\mathcal{R}_{q+r}^{(s)}=\pi_{q+r}^{q+r+s}\left(\mathcal{R}_{q+r+s}\right)$ is of higher order than the original one; a concrete example for this effect will be given in Sect. 6 . The proof of the Cartan-Kuranishi Theorem even provides a concrete algorithm for the completion to an involutive equation. We briefly sketch it here; it requires the (geometric) symbol $\mathcal{G}_{q}=V_{q-1}\left(\mathcal{R}_{q}\right) \subseteq V_{q-1}\left(J_{q} \mathcal{M}\right)$ of a differential equation $\mathcal{R}_{q} \subseteq J_{q} \mathcal{M}$ where $V_{q-1}\left(J_{q} \mathcal{M}\right)=\operatorname{ker} T \pi_{q-1}^{q} \subset T J_{q} \mathcal{M}$. We cannot detail this construction here; especially, we cannot explain what an involutive symbol is. For all these details we refer again to the literature cited above.

The completion algorithm consists essentially of two nested loops. At the beginning we set $s=r=0$. In the inner loop we prolong the equation, i. e. we increase $r$, until we reach an equation with an involutive symbol. One can show that this always happens after a finite number of prolongations. The thus obtained equation $\mathcal{R}_{q+r}^{(s)}$ is prolonged once more and then projected back, i. e. we determine $\mathcal{R}_{q+r}^{(s+1)}$. If the two manifolds are 
equal, we stop, as then $\mathcal{R}_{q+r}^{(s)}$ is the sought involutive equation. Otherwise we increase $s$ and iterate. By a Noetherian argument the algorithm terminates.

In the special case of ordinary differential equations, in which we are mostly interested in this article, this completion algorithm can be considerably simplified. The inner loop is no longer necessary, as the symbol of an ordinary differential equation is always involutive. This implies that the final equation has always the same order as the original one. Furthermore, we can reverse the order of the operations in the algorithm. In the general case we always first prolong and then project; for ordinary differential equations it suffices to first project, i. e. to explicitly exhibit the constraint manifold, and then to prolong just this manifold. Thus we construct, starting with $\mathcal{R}_{q}^{(0)}=\mathcal{R}_{q}$, the sequence of differential equations

$$
\mathcal{R}_{q}^{(s+1)}=\mathcal{R}_{q}^{(s)} \cap J_{1} \mathcal{R}_{q-1}^{(s+1)},
$$

until we find $\mathcal{R}_{q}^{(s+1)}=\mathcal{R}_{q}^{(s)}$. This happens at the latest after $\operatorname{dim} \mathcal{R}_{q}$ iterations.

This difference between ordinary and partial differential equations has a very simple explanation. In a system of ordinary differential equations only one mechanism for the generation of integrability conditions exists: the system contains equations of differing orders and the prolongation of the lower order ones leads to new equations. These equations of lower order describe the constraint manifold $\mathcal{R}_{q-1}^{(s+1)}$ on the right hand side of (4); its prolongation is $J_{1} \mathcal{R}_{q-1}^{(s+1)}$.

In a system of partial differential equations a second mechanism exists, a generalisation of the classical cross-derivative: we take a linear combination of prolonged equations; if the linear combination is such that all derivatives of maximal order cancel, we obtain an integrability condition of lower order. Obviously, it is crucial here that we first prolong and then project; otherwise we might overlook integrability conditions. As simple examples like $u_{z z}+y u_{x x}=0=u_{y y}$ demonstrate, we might need several prolongations for the cross-derivative and the integrability conditions (here $u_{x x y}=0$ and $u_{x x x x}=0$ ) might be of higher order than the original system. Involution of the symbol $\mathcal{G}_{q}$ is concerned with the maximal number of prolongations needed.

\section{Time-dependent Hamiltonian Systems and Cosymplectic Manifolds}

In the literature one can find a number of approaches for dealing with time-dependent Hamiltonian systems. The most convenient one for our purposes uses cosymplectic geometry, an extension of symplectic geometry to odd-dimensional manifolds. As we will see below, a cosymplectic manifold provides us in a natural way with a clock.

Definition 4. A cosymplectic manifold is a $(2 n+1)$-dimensional manifold $\mathcal{M}$ equipped with a closed 2-form $\Omega$ and a closed 1-form $\eta$ such that $\Omega^{n} \wedge \eta$ is a volume form.

The cosymplectic structure induces the vector bundle isomorphism [1]

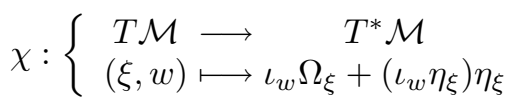


generalising the musical isomorphism $b$ on a symplectic manifold. Furthermore, there exists a unique vector field $R$ on $\mathcal{M}$, the Reeb vector field, such that $\iota_{R} \Omega=0$ and $\iota_{R} \eta=1$; it is given by $R=\chi^{-1}(\eta)$. The Darboux theorem for cosymplectic manifolds asserts that locally there always exists a coordinate system $\left(q^{i}, p_{i}, t\right)$ such that $\Omega=d q^{i} \wedge d p_{i}, \eta=d t$ (and hence $R=\partial_{t}$ ).

EXAMPLE 1. Every symplectic manifold can trivially be extended to a cosymplectic manifold as follows. Let $(\mathcal{M}, \Omega)$ be a symplectic manifold and $d t$ the canonical one-form on $\mathbb{R}$. Then the product manifold $\mathcal{M} \times \mathbb{R}$ together with the forms $p r_{1}^{*} \Omega$ and $p r_{2}^{*} d t$ (here $p r_{i}$ denotes the projection on the $i$ th factor) is a cosymplectic manifold.

On a cosymplectic manifold we associate three different types of "gradient fields" [2] with a function $H: \mathcal{M} \rightarrow \mathbb{R}$ :

1. Cosymplectic gradient: $\operatorname{grad} H=\chi^{-1}(d H)=H_{t} \partial_{t}+H_{p_{i}} \partial_{q^{i}}-H_{q^{i}} \partial_{p_{i}}$.

2. Hamiltonian vector field: $X_{H}=\chi^{-1}(d H-R(H) \eta)=H_{p_{i}} \partial_{q^{i}}-H_{q^{i}} \partial_{p_{i}}$.

3. Evolution vector field: $E_{H}=R+X_{H}=\partial_{t}+H_{p_{i}} \partial_{q^{i}}-H_{q^{i}} \partial_{p_{i}}$.

One can show that the Hamiltonian vector fields form an involutive distribution. If we assume that $\pi: \mathcal{M} \rightarrow \mathcal{B}$ is a fibred manifold such that $\operatorname{dim} \mathcal{B}=1$ and $T_{\xi} \pi\left(R_{\xi}\right) \neq 0$ for all $\xi \in \mathcal{M}$, i. e. the Reeb vector field $R$ is everywhere transversal to the fibration, then the Hamiltonian vector fields span the vertical bundle $V \mathcal{M}$. Hence $R$ defines the horizontal spaces of a connection on $\mathcal{M}$. The corresponding vertical projector is

$$
\mathcal{V}[R]:\left\{\begin{array}{cc}
T \mathcal{M} & \longrightarrow \mathcal{M} \\
(\xi, w) & \longmapsto\left(\xi, w-\mu R_{\xi}\right)
\end{array}\right.
$$

where the coefficient $\mu \in \mathbb{R}$ is determined by the relation $T_{\xi} \pi(w)=\mu T_{\xi} \pi\left(R_{\xi}\right)$.

One possibility to model a time-dependent Hamiltonian system consists of using a cosymplectic manifold $(\mathcal{M}, \Omega, \eta)$ and a Hamiltonian $H: \mathcal{M} \rightarrow \mathbb{R}$. The manifold $\mathcal{M}$ is often called the extended phase space. The Reeb vector field provides us with an intrinsic clock; ${ }^{1}$ it serves as "reference" for the evolution of the system determined by the evolution vector field $E_{H}$. Trajectories of the system correspond thus to integral curves of $E_{H}$.

Alternatively, we may combine the cosymplectic structure and the Hamiltonian by introducing the 2-form $\Omega^{H}=\Omega+d H \wedge \eta$. One easily checks that $\left(\mathcal{M}, \Omega^{H}, \eta\right)$ is again a cosymplectic manifold. Its Reeb vector field $R^{H}$ is just the evolution vector field $E_{H}$ associated to $H$ with respect to the original cosymplectic structure. This formulation turns out to be convenient for the analysis of systems with constraints.

Assume we are given a time-dependent Hamiltonian system modelled by the cosymplectic manifold $\left(\mathcal{M}, \Omega^{H}, \eta\right)$; constraints correspond to a submanifold $\mathcal{N} \subseteq \mathcal{M}$. It is

\footnotetext{
${ }^{1}$ With this interpretation, it appears natural from a physical point of view to require that the Reeb vector field $R$ is even projectable. This condition is automatically satisfied, if $\eta=\pi^{*} \hat{\eta}$ where $\hat{\eta}$ is a one-form on $\mathcal{B}$, as is the case with most physical applications. However, for our mathematical results this assumption is not necessary.
} 
well-known that a constrained system is not necessarily consistent, as the given constraints may lead to further ones. It is the task of the so-called constraint algorithm to extract all hidden constraints.

This algorithm may be formulated in many different ways but the basic idea is very simple: we require that the vector field $R^{H}$ must be everywhere tangent to the constraint manifold $\mathcal{N}$, as otherwise its integral curves and thus the trajectories of the system cannot stay in $\mathcal{N}$. This leads to a sequence of submanifolds $\mathcal{N}_{s}$ with $\mathcal{N}_{1}=\mathcal{N}$ and

$$
\mathcal{N}_{s+1}=\left\{\xi \in \mathcal{N}_{s} \mid R_{\xi}^{H} \in T_{\xi} \mathcal{N}_{s}\right\}
$$

This description can be applied to arbitrary vector fields, the cosymplectic structure plays no role here. But given this structure, we may reformulate (7) similar to the Gotay-Nester formulation of the Dirac theory [6]. We first introduce the cosymplectic complement $\left(T_{\xi} \mathcal{N}\right)^{\perp}=\left\{w \in T_{\xi} \mathcal{M} \mid\left\langle\chi^{H}(w), T_{\xi} \mathcal{N}\right\rangle=0\right\}$ where $\chi^{H}$ is the bundle isomorphism (5) for the cosymplectic structure given by $\Omega^{H}$ and $\eta$. Then we get [3]

$$
\mathcal{N}_{s+1}=\left\{\xi \in \mathcal{N}_{s} \mid\left\langle\eta_{\xi},\left(T_{\xi} \mathcal{N}_{s}\right)^{\perp}\right\rangle=0\right\}
$$

However, we will not need this formulation in the sequel.

Note that the above description slightly simplifies the situation actually encountered in the modelling of constrained mechanical systems. There the Hamiltonian $H$ is welldefined only on the (primary) constraint manifold $\mathcal{N}$. This may be taken into account by allowing that $H$ depends on parameters. Thus instead of a single vector field $R^{H}$ we are dealing with a whole pencil of vector fields and at each step of the constraint algorithm tangency may now be achieved either by shrinking the pencil or the constraint manifold. In Dirac's terminology we are using the "total Hamiltonian" $H_{T}=H+\mu^{\alpha} \phi_{\alpha}$ where the functions $\phi_{\alpha}$ are the primary constraints defining $\mathcal{N}$.

\section{Hamiltonian Differential Equations}

Let $(\mathcal{M}, \Omega, \eta)$ be a cosymplectic manifold that at the same time is the total space of the fibration $\pi: \mathcal{M} \rightarrow \mathcal{B}$ with $\operatorname{dim} \mathcal{B}=1$. Again we assume that the Reeb vector field $R$ is everywhere transversal to the fibration. Let furthermore $\mathcal{R}_{1} \subseteq J_{1} \mathcal{M}$ be a first order ordinary differential equation. We assume for the moment that we are dealing with a system without constraints, i. e. $\mathcal{R}_{1}=\operatorname{Im} \gamma$ for a section $\gamma: \mathcal{M} \rightarrow J_{1} \mathcal{M}$.

This implies that now we have two connections: one stems from the cosymplectic structure and has as vertical projector $\mathcal{V}[R]$ given by $(6)$; the other one comes from the differential equation and has as vertical projector $\mathcal{V}[\gamma]$ given by (3). We can thus associate with the differential equation $\mathcal{R}_{1}$ the vector field $X$ on $\mathcal{M}$ defined by

$$
X: \xi \longmapsto-\mathcal{V}[\gamma]\left(R_{\xi}\right)=\mathcal{V}[R]\left(\Gamma_{1}\left(\gamma(\xi), T_{\xi} \pi\left(R_{\xi}\right)\right)\right)
$$

$X_{\xi}$ is the difference between the horizontal lifts of the vector $T_{\xi} \pi\left(R_{\xi}\right)$ with respect to the two different connections. Hence $X$ is a vertical field. 
Definition 5. The differential equation $\mathcal{R}_{1} \subseteq J_{1} \mathcal{M}$ is Hamiltonian with respect to the cosymplectic structure on $\mathcal{M}$, if the vector field $X$ defined by (8) is Hamiltonian, i. e. if a function $H: \mathcal{M} \rightarrow \mathbb{R}$ exists such that $X=X_{H}$.

As the Hamiltonian vector fields span the vertical bundle, Darboux coordinates $\left(t, q^{i}, p_{i}\right)$ on the cosymplectic manifold $\mathcal{M}$ are automatically adapted coordinates for the fibred manifold $\pi: \mathcal{M} \rightarrow \mathcal{B}$. In these coordinates, the differential equation $\mathcal{R}_{1}$ is locally represented by equations $\dot{q}^{i}=f^{i}(t, q, p), \dot{p}_{i}=g_{i}(t, q, p)$. The vector field $X$ is then given by $-\mathcal{V}[\gamma]\left(\partial_{t}\right)=f^{i} \partial_{q^{i}}+g_{i} \partial_{p_{i}}$ and we recover that Hamiltonian differential equations have the familiar local form $(1)$.

TheOREM 2. A section $\sigma: \mathcal{B} \rightarrow \mathcal{M}$ is a solution of the Hamiltonian differential equation $\mathcal{R}_{1}$ in the sense of Definition 2, if and only if $\operatorname{Im} \sigma$ is an integral curve of the evolution vector field $E_{H}=R+X_{H}$.

Indeed, it follows from (8) that $\left(E_{H}\right)_{\xi}=\Gamma_{1}\left(\gamma(\xi), T_{\xi} \pi\left(R_{\xi}\right)\right)$. If the section $\sigma$ is a solution, then $\gamma \circ \sigma=j_{1} \sigma$. Thus, by definition of the contact map, we get at the point $\xi=\sigma(x)$ that $\left(E_{H}\right)_{\xi}=T_{x} \sigma \cdot T_{\xi} \pi\left(R_{\xi}\right) \in T_{\xi}(\operatorname{Im} \sigma)$ and $\operatorname{Im} \sigma$ is an integral curve of $E_{H}$. Conversely, it follows from the transversality of $E_{H}$ that every integral curve is (at least locally) the image of a section $\sigma: \mathcal{B} \rightarrow \mathcal{M}$. Applying the same argument backwards, we see that $\gamma \circ \sigma=j_{1} \sigma$.

Next we study the effect of constraints. Assume we are given a cosymplectic manifold $\left(\mathcal{M}, \Omega^{H}, \eta\right)$ and a submanifold $\mathcal{N} \subseteq \mathcal{M}$. With the help of the contact map, the vector field $R^{H}$ uniquely induces a section $\gamma: \mathcal{M} \rightarrow J_{1} \mathcal{M}$ whose image defines a Hamiltonian equation $\overline{\mathcal{R}}_{1}$. The constrained system is then realised by the differential equation

$$
\mathcal{R}_{1}=\left\{(\xi, \lambda) \in \overline{\mathcal{R}}_{1} \mid \xi \in \mathcal{N}\right\} .
$$

Thus $\mathcal{R}_{0}^{(1)}=\mathcal{N}$. Now we are going to compare the cosymplectic constraint algorithm with the generic completion algorithm for differential equations described in Sect. 2 . It leads to a sequence of constraint manifolds $\mathcal{R}_{0}^{(s)}$ such that

$$
\mathcal{R}_{0}^{(s+1)}=\pi_{0}^{1}\left(\mathcal{R}_{1}^{(s-1)} \cap J_{1} \mathcal{R}_{0}^{(s)}\right) \subseteq \mathcal{M} .
$$

THEOREM 3. Both algorithms are equivalent and yield the same sequence of constraint manifolds, i. e. $\mathcal{R}_{0}^{(s)}=\mathcal{N}_{s}$ for all $s \geq 1$.

This theorem is a simple consequence of our definition of the first-order jet bundle. The basic idea is to describe the manifolds $\mathcal{R}_{0}^{(s)}$ in a similar manner as (7). By construction, the equality

$$
\Gamma_{1}\left(\gamma(\xi), T_{\xi} \pi\left(R_{\xi}^{H}\right)\right)=R_{\xi}^{H}
$$

holds. The section $\gamma$ defines what one calls in the numerical analysis literature an underlying differential equation, i. e. a differential equation that for initial data on the constraint manifold has the same solution as our constrained system. In fact, it would suffice, if (9) 
held only for all $\xi \in \mathcal{N}$, i. e. we could take any section such that $\mathcal{R}_{1} \subseteq \operatorname{Im} \gamma$. It is now easy to see that

$$
\mathcal{R}_{0}^{(s+1)}=\left\{\xi \in \mathcal{R}_{0}^{(s)} \mid \gamma(\xi) \in J_{1} \mathcal{R}_{0}^{(s)}\right\} .
$$

This formulation is in close analogy to (7). Assume that $\gamma(\xi)=(\xi, \lambda) \in J_{1} \mathcal{R}_{0}^{(s)}$ which means that $\lambda \in T_{\pi(\xi)}^{*} \mathcal{B} \otimes T_{\xi} \mathcal{R}_{0}^{(s)}$ according to our definition of the jet bundle. Applying (9) shows that this is equivalent to $R_{\xi}^{H} \in T_{\xi} \mathcal{R}_{0}^{(s)}$, as for such a $\lambda$ the contact map $\Gamma_{1}$ maps to the tangent space $T_{\xi} \mathcal{R}_{0}^{(s)}$. Hence $\xi \in \mathcal{R}_{0}^{(s)}$ implies $\xi \in \mathcal{N}_{s}$ and vice versa.

It is easy to see that the theorem remains true, if we are dealing with a parametrised Hamiltonian as discussed at the end of Sect. 3. In this case the section $\gamma$ is parametrised, too, and we always encounter the same conditions on either the parameters or the constraint manifolds. Note that for our theorem the cosymplectic structure is actually irrelevant; instead of the vector field $R^{H}$ we could have taken any vector field. What we have proven is the general equivalence of the completion in the jet bundle and in the tangent bundle. As mentioned in the introduction, the latter one has already been studied by many authors.

\section{Covariant Classical Mechanics}

As a larger example of our approach we consider covariant classical mechanics $[7,8]$. It leads naturally to the geometric structures considered in this article. The theory postulates six fundamental geometric objects: (i) an oriented four-dimensional manifold, the classical spacetime $\mathcal{E}$; (ii) an oriented one-dimensional affine space modelled on a vector space $\mathbb{T}$, the absolute time $\mathcal{T}$; (iii) a surjective submersion $t: \mathcal{E} \rightarrow \mathcal{T}$, the time fibration; (iv) a scaled Riemannian metric on the fibres of spacetime, the spacelike metric $g$; (v) a linear connection on spacetime that preserves the time fibration and the spacelike metric and whose curvature has the symmetries of Riemannian connections, the gravitational field $K^{\natural}$ and (vi) a scaled closed 2-form on the spacetime, the electromagnetic field $f$. "Scaled" means that these objects are tensorialised by a suitable scale factor accounting for the appropriate units of measurement. In particular, $\mathbb{T}$ denotes the vector space associated with time units. As phase space for a classical particle we take the first-order jet space $J_{1} \mathcal{E}$ over the spacetime fibration; it plays the role of the fibred manifold $\mathcal{M}$ in the previous sections.

Note that we assume a fibration of spacetime over time, but, according to the principle of general relativity, not a distinguished splitting of spacetime into space and time. An observer is a section $o: \mathcal{E} \rightarrow J_{1} \mathcal{E}$ and thus defines a connection on spacetime inducing such a splitting. Consequently, we do not assume the existence of distinguished observers.

The six above postulated objects induce in a covariant way two further objects $[7,8]$ : the time form $d t: \mathcal{E} \rightarrow \mathbb{T} \otimes T^{*} \mathcal{E}$ on the spacetime and a 2-form $\Omega^{\natural}: J_{1} \mathcal{E} \rightarrow \Lambda^{2}\left(T^{*} J_{1} \mathcal{E}\right)$ on the phase space. Here, the symbol ${ }^{\natural}$ denotes objects derived from the gravitational field. The pair $\left(\Omega^{\natural}, d t\right)$ defines a scaled cosymplectic structure on the phase space [16].

Given a particle of mass $m$ and charge $q$, we can get rid of any choice of length and mass units of measurement by "normalising" the spacelike metric and the electromagnetic field. Accordingly, all objects derived from the normalised objects include the 
mass and the charge of the particle. It is now convenient to add the gravitational and electromagnetic objects in a covariant way according to the formula $\Omega=\Omega^{\natural}+F$ where $F$ is the normalised electromagnetic field. The total 2 -form $\Omega$ encodes the full structure of spacetime (metric, gravitational and electromagnetic field) and hence plays a central role in the theory. Moreover, $\left(J_{1} \mathcal{E}, \Omega, d t\right)$ is a scaled cosymplectic manifold [16].

Classical mechanics can now be described as follows. The scaled cosymplectic structure yields a scaled Reeb vector field $\varrho: J_{1} \mathcal{E} \rightarrow \mathbb{T}^{*} \otimes T J_{1} \mathcal{E}$. Because of the scaling, the field $\varrho$ may be interpreted as a section $J_{1} \mathcal{E} \rightarrow J_{1}\left(J_{1} \mathcal{E}\right)$ (we have $T^{*} \mathcal{T} \cong \mathcal{T} \otimes \mathbb{T}^{*}$ ). Thus, $\operatorname{Im} \varrho$ is a differential equation over $\mathcal{M}=J_{1} \mathcal{E}$ and the two approaches, vector field on a cosymplectic manifold or differential equation in a jet bundle, coincide!

Of course, by construction, Im $\varrho$ is a Hamiltonian differential equation in the sense of Definition 5 with respect to the cosymplectic structure $(\Omega, d t)$ for the Hamiltonian $H \equiv 0$. But obviously, this is not a very interesting point of view. Hamiltonian dynamics is concerned with the time evolution on the spacetime. Thus we need an observer $o$ in order to embed $\mathcal{T}$ in the spacetime $\mathcal{E}$.

As an observer $o$ is a section $\mathcal{E} \rightarrow J_{1} \mathcal{E}$, its first prolongation $o^{(1)}$ is the unique map $J_{1} \mathcal{E} \rightarrow J_{1}\left(J_{1} \mathcal{E}\right)$ satisfying $o^{(1)} \circ j_{1} \sigma=j_{1}(o \circ \sigma)$ for all sections $\sigma: \mathcal{T} \rightarrow \mathcal{E}$. However, $o^{(1)}$ is not a section; but it becomes a section, if we compose it with a certain natural involution $r: J_{1}\left(J_{1} \mathcal{E}\right) \rightarrow J_{1}\left(J_{1} \mathcal{E}\right)[9]{ }^{1}$ Thus $r \circ o^{(1)}$ defines a connection on $J_{1} \mathcal{E}$ which may be used to split, at least locally, the total 2 -form $\Omega$ into two parts. Indeed, locally a scaled function $H_{o}: J_{1} \mathcal{E} \rightarrow \mathbb{T}^{*} \otimes \mathbb{R}$ exists such that the scaled vector field $\varrho_{o}=\varrho-X_{H_{o}}$ spans the horizontal distribution of the connection defined by $r \circ o^{(1)}$. It is easy to check that the 2-form $\Omega_{o}=\Omega-d H_{o} \wedge d t$ (here we naturally identify $d H_{o} \wedge d t$ with an unscaled 2 -form via the pairing of $\mathbb{T}^{*}$ and $\mathbb{T}$ ) defines together with $d t$ an alternative cosymplectic structure on $J_{1} \mathcal{E}$ and $\varrho_{o}$ is the corresponding Reeb vector field.

We may write $\Omega=\Omega_{o}^{H_{o}}$ with the notation introduced in Section 3. For any choice of the observer $o, \operatorname{Im} \varrho$ is a Hamiltonian differential equation with respect to the cosymplectic structure $\left(\Omega_{o}, d t\right)$ and the Hamiltonian $H_{o}$. The vector field $\varrho$ is obviously the evolution vector field $E_{H_{o}}=\varrho_{o}+X_{H_{o}}$.

\section{Field Theories}

We already mentioned above that the completion of partial differential equations is more involved than that of ordinary differential equations. This obviously affects the generalisation of our results to field theories. Instead of discussing such an extension, we only present two examples of "pathological" Lagrangians exhibiting some of the arising problems. Both examples are completely artificial and do not lead to meaningful physical theories. However, from a mathematical point of view they represent valid Lagrangians and any constraint theory for field theories should be able to handle them.

The first example shows that naive generalisations of the completion theory for ordinary differential equations do not suffice for checking the consistency of a theory. Such approaches have a long tradition in the physical literature; already Dirac [4] applied one

\footnotetext{
${ }^{1}$ This is exactly the same problem as with the tangent map of a vector field which defines a vector field on the tangent bundle only after the swapping of certain components.
} 
in his analysis of a Hamiltonian form of the Maxwell equations. He simply followed the same strategy as in the finite-dimensional case: always compute the Poisson brackets of the constraints with the Hamiltonian. However, taking Poisson brackets corresponds to computing time derivatives. Thus this approach can only find integrability conditions arising from cross-derivatives including at least one time derivative; integrability conditions arising from purely spatial cross-derivatives are not detected.

Let the fields $\phi, \lambda$ and $\mu$ be defined on a $(2+1)$-dimensional space-time with coordinates $(x, y, t)$ and consider the family of Lagrangian densities

$$
\mathcal{L}[\phi, \lambda, \mu]=\frac{1}{2} \phi_{t}^{2}+\lambda\left(\phi_{x}-F(\phi)\right)+\mu\left(\phi_{y}-G(\phi)\right)
$$

where $F$ and $G$ are yet arbitrary functions. It leads to the Euler-Lagrange equations

$$
\begin{gathered}
\phi_{t t}+\lambda_{x}+\mu_{y}+\lambda F^{\prime}(\phi)+\mu G^{\prime}(\phi)=0, \\
\phi_{x}-F(\phi)=0, \quad \phi_{y}-G(\phi)=0 .
\end{gathered}
$$

An obvious integrability condition of this system is $F^{\prime}(\phi) G(\phi)=F(\phi) G^{\prime}(\phi)$. If it is not automatically satisfied due to the properties of the functions $F$ and $G$, the theory is either inconsistent or admits only a constant field $\phi$ as solution. This condition arises from a purely spatial cross-derivative of the equations in (11); no time derivative is involved.

It is easy to check that using the Dirac approach one cannot find this condition [21] and thus possibly fails to notice that one is working with an inconsistent theory. This example clearly demonstrates the importance of using a covariant approach that treats differentiations with respect to time and space in the same way.

The second example concerns the problem that the completion may lead to higherorder equations; it shows that this is not only a theoretical possibility but actually may happen in systems derived from variational principles. In such a situation it becomes very difficult to give a Hamiltonian interpretation of the field equations.

With the same fields as above, we study the Lagrangian density

$$
\mathcal{L}[\phi, \lambda, \mu]=\frac{1}{2} \phi_{t}^{2}+\frac{1}{2} y \phi_{x}^{2}+\lambda_{y} \phi_{y}+\lambda \mu .
$$

Its Euler-Lagrange equations are

$$
\begin{aligned}
& \phi_{t t}+y \phi_{x x}+\lambda_{y y}=0, \\
& \phi_{y y}-\mu=0, \quad \lambda=0 .
\end{aligned}
$$

Without going into any details, we state that applying the above outlined CartanKuranishi completion algorithm leads after six prolongations and four projections to a formally integrable fourth-order system:

$$
\begin{gathered}
\phi_{t t}+y \phi_{x x}=0, \quad \phi_{y y}-\mu=0, \quad \lambda=0 \\
2 \phi_{x x y}+y \mu_{x x}+\mu_{t t}=0, \quad \mu_{y t t}+y \mu_{x x t}+3 \mu_{x x}=0 \\
\mu_{t t t t}-2 \phi_{x x x x}+2 y \mu_{x x t t}+y^{2} \mu_{x x x x}=0 .
\end{gathered}
$$


Note in particular that (14) is of fourth order in time! Rewriting the Euler-Lagrange equations as a Hamiltonian system via a Legendre transformation does not change anything; one obtains a similar rise in order.

\section{Conclusions}

The goal of this article was to unify differential equation theory and geometric mechanics in an intrinsic manner. We have shown that this is possible for finite-dimensional Hamiltonian systems using cosymplectic manifolds. As an additional benefit the arising theory handles non-autonomous systems without the introduction of any artificial constructions like an extended configuration space.

The natural next step consists of extending this approach to field theories. As we indicated in the last section based on two examples, completely new problems emerge. Some of them can probably be resolved in a multi-symplectic framework, but in particular the interpretation of systems of higher order remains a puzzle. We want to stress again that although both examples are completely artificial, any constraint algorithm for field theories must either be able to handle them or at least detect that they are pathological. The popular naive generalisations of the Dirac theory achieve neither.

\section{Acknowledgements}

The authors thank Manuel de León and Marco Modugno for a number of interesting discussions. The work of WMS has been supported by Deutsche Forschungsgemeinschaft and by INTAS (grant 99-1222). He greatly appreciates the invitation by the organisers of the 33rd Symposium on Mathematical Physics in Torun to present this material at the conference.

\section{REFERENCES}

[1] C. Albert. Le théorème de réduction de Marsden-Weinstein en géométrie cosymplectique et de contact. J. Geom. Phys., 6:627-649, 1989.

[2] F. Cantrijn, M. de León, and E.A. Lacomba. Gradient vector fields on cosymplectic manifolds. J. Phys. A: Math. Gen., 25:175-188, 1992.

[3] D. Chinea, M. de León, and J.C. Marrero. The constraint algorithm for time-dependent Lagrangians. J. Math. Phys., 35:3410-3447, 1994.

[4] P.A.M. Dirac. Lectures on Quantum Mechanics. Belfer Graduate School Monograph Series 3. Yeshiva University, New York, 1964.

[5] M. Dubois-Violette. The theory of overdetermined linear systems and its applications to non-linear field equations. J. Geom. Phys., 1:139-172, 1984.

[6] M.J. Gotay, J.M. Nester, and G. Hinds. Presymplectic manifolds and the Dirac-Bergmann theory of constraints. J. Math. Phys., 19:2388-2399, 1978.

[7] A. Jadcyk, J. Janyška, and M. Modugno. Galilei general relativistic quantum mechanics revisited. In A.S. Alves, F.J. Craveiro de Carvalho, and J.A. Pereira da Silva, editors, Geometria, Física-Matemática e Outros Ensaios, pages 253-313. 1998. 
[8] A. Jadcyk and M. Modugno. Galilei general relativistic quantum mechanics. Technical report, Dept. of Applied Mathematics, University of Florence, 1994.

[9] L. Mangiarotti and M. Modugno. New operations on jet spaces. Ann. Fac. Science Toulouse, Serie V, 5:171-198, 1983.

[10] G. Marmo, G. Mendella, and W.M. Tulczyjew. Constrained Hamiltonian systems as implicit differential equations. J. Phys. A: Math. Gen., 30:277-294, 1996.

[11] G. Mendella, G. Marmo, and W.M. Tulczyjew. Integrability of implicit differential equations. J. Phys. A: Math. Gen., 28:149-163, 1995.

[12] M.C. Muñoz-Lecanda and N. Román-Roy. Implicit quasilinear differential systems: A geometrical approach. Electr. J. Diff. Eqs., 1999. Paper No. 10.

[13] J.F. Pommaret. Systems of Partial Differential Equations and Lie Pseudogroups. Gordon \& Breach, London, 1978.

[14] P.J. Rabier and W.C. Rheinboldt. A geometric treatment of implicit differential algebraic equations. J. Diff. Eq., 109:110-146, 1994.

[15] S. Reich. On an existence and uniqueness theory for nonlinear differential-algebraic equations. Circ. Sys. Sig. Proc., 10:343-359, 1991.

[16] D. Saller and R. Vitolo. Symmetries in covariant classical mechanics. J. Math. Phys., 41:6824-6842, 2000.

[17] D.J. Saunders. The Geometry of Jet Bundles. London Mathematical Society Lecture Notes Series 142. Cambridge University Press, Cambridge, 1989.

[18] W.M. Seiler. Analysis and Application of the Formal Theory of Partial Differential Equations. PhD thesis, School of Physics and Materials, Lancaster University, 1994.

[19] W.M. Seiler. Involution and constrained dynamics II: The Faddeev-Jackiw approach. J. Phys. A: Math. Gen., 28:7315-7331, 1995.

[20] W.M. Seiler. Involution and constrained dynamics III: Intrinsic degrees of freedom count. Tech. Mech., 20:137-146, 2000.

[21] W.M. Seiler and R.W. Tucker. Involution and constrained dynamics I: The Dirac approach. J. Phys. A: Math. Gen., 28:4431-4451, 1995.

[22] A. Szatkowski. Geometric characterization of singular differential algebraic equations. Int. J. Sys. Sci., 23:167-186, 1992. 